\title{
Article
}

\section{0-S Retrogated Compressed Sensing 2D Cine of the Heart: Sharper Borders and Accurate Quantification}

\author{
Benjamin Longère ${ }^{1, *}\left(\mathbb{0}\right.$, Christos V. Gkizas ${ }^{2}$, Augustin Coisne ${ }^{1}\left(\mathbb{D}\right.$, Lucas Grenier $^{2}$, Valentina Silvestri ${ }^{2}$, \\ Julien Pagniez ${ }^{2}$, Arianna Simeone ${ }^{2}$, Justin Hennicaux ${ }^{2}$, Michaela Schmidt ${ }^{3}$, Christoph Forman ${ }^{3}{ }^{1}$, \\ Solenn Toupin ${ }^{4}$, David Montaigne ${ }^{1}$ (D) and François Pontana ${ }^{1}$ (I)
}

check for

updates

Citation: Longère, B.; Gkizas, C.V.; Coisne, A.; Grenier, L.; Silvestri, V.; Pagniez, J.; Simeone, A.; Hennicaux, J.; Schmidt, M.; Forman, C.; et al. 60-S Retrogated Compressed Sensing 2D Cine of the Heart: Sharper Borders and Accurate Quantification. J. Clin Med. 2021, 10, 2417. https://doi.org/ $10.3390 / \mathrm{jcm} 10112417$

Academic Editor: Mickaël Ohana

Received: 16 March 2021

Accepted: 26 May 2021

Published: 29 May 2021

Publisher's Note: MDPI stays neutral with regard to jurisdictional claims in published maps and institutional affiliations.

Copyright: (c) 2021 by the authors. Licensee MDPI, Basel, Switzerland. This article is an open access article distributed under the terms and conditions of the Creative Commons Attribution (CC BY) license (https:/ / creativecommons.org/licenses/by/ $4.0 /)$.
1 University of Lille, Inserm, CHU Lille, Institut Pasteur Lille, U1011-European Genomic Institute for Diabetes (EGID), F-59000 Lille, France; augustin.coisne@chru-lille.fr (A.C.); david.montaigne@chru-lille.fr (D.M.); francois.pontana@chru-lille.fr (F.P.)

2 CHU Lille, Department of Cardiovascular Radiology, F-59000 Lille, France; chgkizas@gmail.com (C.V.G.); lucas.grenier@chru-lille.fr (L.G.); valentina.silvestri@chru-lille.fr (V.S.); julien.pagniez@chru-lille.fr (J.P.); arianna.simeone@chru-lille.fr (A.S.); justin.hennicaux@chru-lille.fr (J.H.)

3 MR Product Innovation and Definition, Magnetic Resonance, Siemens Healthcare GmbH 91052 Erlangen, Germany; michaela.schmidt@siemens-healthineers.com (M.S.); christoph.forman@siemens-healthineers.com (C.F.)

4 Scientific Partnerships, Siemens Healthcare France, 93200 Saint-Denis, France; solenn.toupin@siemens-healthineers.com

* Correspondence: benjamin.longere@chru-lille.fr

Abstract: Background and objective: Real-time compressed sensing cine $\left(\mathrm{CS}_{\mathrm{rt}}\right)$ provides reliable quantification for both ventricles but may alter image quality. The aim of this study was to assess image quality and the accuracy of left (LV) and right ventricular (RV) volumes, ejection fraction and mass quantifications based on a retrogated segmented compressed sensing $2 \mathrm{D}$ cine sequence $\left(\mathrm{CS}_{\mathrm{rg}}\right)$. Methods: Thirty patients were enrolled. Each patient underwent the reference retrogated segmented steady-state free precession cine sequence $\left(\mathrm{SSFP}_{\text {ref }}\right)$, the real-time $\mathrm{CS}_{\mathrm{rt}}$ cine and the segmented retrogated prototype $\mathrm{CS}_{\mathrm{rg}}$ sequence providing the same slices. Functional parameters quantification and image quality rating were performed on $\mathrm{SSFP}_{\text {ref }}$ and $\mathrm{CS}_{\mathrm{rg}}$ images sets. The edge sharpness, which is an estimate of the edge spread function, was assessed for the three sequences. Results: The mean scan time was: $\mathrm{SSFP}_{\text {ref }}=485.4 \pm 83.3(\mathrm{SD}) \mathrm{s}(95 \% \mathrm{CI}: 454.3-516.5)$ and $\mathrm{CS}_{\mathrm{rg}}=58.3 \pm 15.1$ (SD) s (95\% CI: 53.7-64.2) ( $p<0.0001)$. CS $_{\text {rg }}$ subjective image quality score (median: 4; range: $\left.2-4\right)$ was higher than the one provided by $\mathrm{CS}_{\mathrm{rt}}$ (median: 3; range: $2-4 ; p=0.0008$ ) and not different from $\mathrm{SSFP}_{\text {ref }}$ overall quality score (median: 4 ; range: $2-4 ; p=0.31$ ). $\mathrm{CS}_{\mathrm{rg}}$ provided similar LV and RV functional parameters to those assessed with $\operatorname{SSFP}_{\text {ref }}(p>0.05)$. Edge sharpness was significantly better with $\mathrm{CS}_{\mathrm{rg}}\left(0.083 \pm 0.013\right.$ (SD) pixel ${ }^{-1} ; 95 \% \mathrm{CI}$ : 0.078-0.087) than with $\mathrm{CS}_{\mathrm{rt}}(0.070 \pm 0.011$ (SD) pixel $^{-1} ; 95 \%$ CI: $\left.0.066-0.074 ; p=0.0004\right)$ and not different from the reference technique $(0.075 \pm 0.016$ (SD) pixel ${ }^{-1}$; 95\% CI: 0.069-0.081; $\left.p=0.0516\right)$. Conclusions: CS $_{\text {rg }}$ cine provides in one minute an accurate quantification of LV and RV functional parameters without compromising subjective and objective image quality.

Keywords: cardiac; heart; magnetic resonance; CMR; compressed sensing; fast imaging; function; retrospective; retrogating; image quality

\section{Introduction}

Cardiac magnetic resonance (CMR) is the reference standard method for quantification of volumes, ejection fraction (EF) and mass of left (LV) and right ventricles (RV) [1-3]. Reliable volumes assessment is required since EF has a strong prognostic value regarding clinical outcomes and survival [4-6]. However, besides steady-state free precession cine images essential for quantification, phase contrast angiography, gadolinium enhanced imaging, and additional sequences may be recommended depending on heart conditions, 
leading to an extended scan time, which may be difficult to handle for patients suffering from cardiac-related shortness of breath, since multiple breath-holdings are required for the acquisition [7].

To reduce acquisition time, compressed sensing (CS) has recently been applied to magnetic resonance imaging, especially CMR [8]. Based on Candès et al.'s work on signal recovery from incomplete sampling, Donoho proposed the CS acquisition $[9,10]$. Since the signal compression required for archiving and transfer implies the deletion of most acquired data, the principle of CS is to acquire only the pieces of information that would be preserved after this compression, sparing the time necessary for the acquisition of the data that would finally be purged during this process. Three prerequisites are mandatory for CS [11]. First, contrary to most medical images, the signal must be compressible. This means a sparsifying transform is required for most transformed coefficients to be insignificant, making the transformed image compressible. Second, the undersampling must be incoherent to provide noise-like overfolding and avoid ambiguity. Finally, nonlinear iterative reconstructions enforcing image consistency with measured signal and transformed image sparsity are needed. In the case of cine imaging, the redundancy in the cardiac cycle provides a strong spatiotemporal correlation, which can be exploited for additional acceleration [12-14]. Undersampling is not only performed in plane but also in the temporal domain by using variable sampling density maps for successive frames.

The first-generation real-time $\mathrm{CS}$ sequence $\left(\mathrm{CS}_{\mathrm{rt}}\right)$ has already been widely evaluated regarding volumes, EF and mass for both ventricles, at 1.5 and 3-Tesla [15-25]. These studies used 80 iterations to reconstruct $\mathrm{CS}_{\mathrm{rt}}$ data, this setting being supposed to provide the best compromise between image quality and reconstruction time [17]. Nevertheless, computing times over one minute per slice were reported, making such a setting not compatible with clinical use [26,27]. Vermersch et al. evaluated the same sequence halving the number of iterations and demonstrated a similar agreement for the quantification of volumes, $\mathrm{EF}$ and mass quantification than previously reported in the literature, extending the use of $\mathrm{CS}_{\mathrm{rt}}$ in clinical practice [22]. To provide an acceleration factor compatible with single-breath-hold real-time imaging, additional sensitivity encoding imaging (SENSE) and partial Fourier are implemented, the latter being a potential cause of edge sharpness impairment [28]. Another limitation is the limited temporal resolution of $49 \mathrm{~ms}$ which may provide variable numbers of frames per slice in the case of heart rate variability. To allow post-processing, a 25-frame per slice interpolation is performed. Besides the border blurring induced by this normalization, end-systolic volumes may be overestimated in the case of a fast heart rate $[29,30]$.

A new generation of retrogated CS sequence $\left(\mathrm{CS}_{\mathrm{rg}}\right)$ has been released. It features segmented acquisition to improve temporal resolution and partial Fourier switch-off to improve edge sharpness. The purpose of this study was to assess the image quality and the ventricular functional parameters in comparison with $\mathrm{CS}_{\mathrm{rt}}$ and the reference retrogated segmented steady-state free precession cine sequence $\left(\mathrm{SSFP}_{\text {ref }}\right)$.

\section{Materials and Methods}

\subsection{Study Population}

From March to April 2019, 30 consecutive adult patients referred for rest CMR were included. Exclusion criteria were grown-up congenital heart disease work-up or follow-up, underaged patients, patients suffering from arrhythmia for whom the use of prospective ECG gating was necessary, MRI contraindications and patient refusal. Patients gave informed consent, and the protocol was approved by our Institutional Ethics Committee.

\subsection{Imaging Protocol}

CMR studies were performed on a 1.5-T scanner (MAGNETOM Aera, Siemens Healthcare, Erlangen, Germany). Every patient underwent three series of cine images: first the reference retrogated segmented multi-breath-hold SSFP sequence $\left(\mathrm{SSFP}_{\text {ref }}\right)$; then the CS-accelerated SSFP real-time sequence $\left(\mathrm{CS}_{\mathrm{rt}}\right)$ acquired in two breath-holds, and finally 
the retrogated segmented SSFP prototype with CS-fashioned acceleration requiring three breath-holds $\left(\mathrm{CS}_{\mathrm{rg}}\right)$. One LV 2-chamber, one RV 2-chamber, one 4-chamber slice and a LV short-axis stack covering the entire ventricles were acquired with the three above-cited sequences, providing identical slice position, thickness, and number. Imaging parameters for the three sequences are summarized in Table 1.

Table 1. Imaging parameters of the reference steady-state free precession cine, real-time compressed sensing cine and segmented retrogated compressed sensing cine.

\begin{tabular}{|c|c|c|c|}
\hline Parameters & SSFP $_{\text {ref }}$ & $\mathrm{CS}_{\mathrm{rt}}$ & $\mathrm{CS}_{\mathrm{rg}}$ \\
\hline Repetition time-ms & 3.16 & 2.70 & 2.70 \\
\hline Echo time-ms & 1.23 & 1.14 & 1.14 \\
\hline Flip angle-degrees & 57 & 60 & 60 \\
\hline Field of view $-\mathrm{mm}^{2}$ & $375 \times 280$ & $360 \times 270$ & $360 \times 270$ \\
\hline Matrix-pixels ${ }^{2}$ & $288 \times 216$ & $224 \times 168$ & $224 \times 168$ \\
\hline Spatial resolution- $\mathrm{mm}^{2}$ & $1.3 \times 1.3$ & $1.6 \times 1.6$ & $1.6 \times 1.6$ \\
\hline Temporal resolution-ms & 37 & 49 & 37 \\
\hline Slice thickness /gap-mm & $8 / 2$ & $8 / 2$ & $8 / 2$ \\
\hline Bandwidth-Hz/pixel & 915 & 900 & 900 \\
\hline Reconstructed cardiac phases- $n$ & - & $25^{\mathrm{a}}$ & - \\
\hline Number of acquired cardiac phases & $25^{\mathrm{a}}$ & $16.8 \pm 3.9$ & $25^{\mathrm{a}}$ \\
\hline Number of breath-holds & $15.0 \pm 1.2$ & $2^{a}$ & $3^{a}$ \\
\hline Cycles per slice $-n$ & $8^{a}$ & $1^{\mathrm{a}}$ & $2^{a}$ \\
\hline Cycles of iterative reconstruction $-n$ & - & 40 & 40 \\
\hline
\end{tabular}

Data are expressed as mean \pm standard deviation in the absence of any indication. ${ }^{a}$ Constant value. Abbreviations: SSFP ref, reference steady-state free precession cine; $\mathrm{CS}_{\mathrm{rt}}$, real-time compressed sensing cine; $\mathrm{CS}_{\mathrm{rg}}$, segmented retrogated compressed sensing cine.

\subsection{Cine Images Quality Assessment}

A 3-step evaluation was performed for the three sequences.

First, a subjective overall image quality was assessed using a 4-point Likert scale (1: non diagnostic, 2: fair, 3: good, 4: excellent).

Then acquisition quality was evaluated using a standardized score based on the " $L V$ function cine SSFP" section of the criteria established from the European CMR registry, evaluating the artifact detection [15] (p. 3). This score was modified, removing its four last items since their score was systematically null, accordingly to our center practice (Table 2). This score increased with acquisition impairment.

Table 2. "LV-Function cine SSFP" section of the standardized objective quality criteria score based on the European CMR registry. Adapted from [15] (p. 3).

\begin{tabular}{|c|c|c|c|c|c|}
\hline Items & 0 & 1 & 2 & 3 & Maximum Score \\
\hline 1. $L V$ coverage & Full & - & No apex & $\begin{array}{c}\text { Base or } \geq 1 \text { slice } \\
\text { missing }\end{array}$ & 5 \\
\hline 2. Wrap around & No & 1 slice & 2 slices & $\geq 3$ slices & \\
\hline 3. Respiratory ghost & No & 1 slice & 2 slices & $\geq 3$ slices & \\
\hline 4. Cardiac ghost & No & 1 slice & 2 slices & $\geq 3$ slices & \\
\hline 5. Blurring/Mistriggering & No & 1 slice & 2 slices & $\geq 3$ slices & 3 \\
\hline 6. Metallic artifacts & No & 1 slice & 2 slices & $\geq 3$ slices & \\
\hline 7. Shimming artifacts & No & 1 slice & 2 slices & $\geq 3$ slices & \\
\hline 8. Signal loss (coil inactive) & Activated & - & Not activated & & 2 \\
\hline 9. Orientation of stack & Correct & - & Incorrect & - & 2 \\
\hline 10. Slice thickness & $\leq 10 \mathrm{~mm}$ & $11-15 \mathrm{~mm}$ & - & $>15 \mathrm{~mm}$ & 3 \\
\hline 11. Gap & $\leq 3 \mathrm{~mm}$ & $3-4 \mathrm{~mm}$ & - & $>4 \mathrm{~mm}$ & 3 \\
\hline 12. Correct LV long axes & $\geq 2 \mathrm{~mm}$ & 1 & - & None & 3 \\
\hline
\end{tabular}


Table 2. Cont.

\begin{tabular}{cccccc}
\hline Items & $\mathbf{0}$ & $\mathbf{1}$ & $\mathbf{2}$ & $\mathbf{3}$ & Maximum Score \\
\hline Score & & & 21 \\
\hline Modified score (items 1 to 8) & & & $\mathbf{1 0}$ \\
\hline
\end{tabular}

The four last items were nulled since acquisitions were repeated when orientation was not appropriated (item $9=0$ ); all acquisitions were performed using a 8-mm thickness (item 10 score $=0$ ) and a 2-mm gap (item 11 score $=0$ ), horizontal and vertical long axis views were systematically acquired (item 12 score $=0$ ). Consequently, italic criteria were not applied, and only bold criteria were used for objective quality assessment in our study, providing a maximum score of 10 points. The more artifacts there were, the higher the score was. Abbreviations: LV, left ventricle; SSFP, steady-state free precession.

Finally, the edge sharpness $(\varepsilon)$ between myocardium and LV blood pool was measured on end-diastole 4-chamber view (Figure 1). This assessment was performed using a MATLAB (version R2015a, The MathWorks, Natick, MA, USA) homemade script. An intensity profile line was drawn perpendicularly to the mid-cavity interventricular septum border with the LV blood pool at end-diastole [31-33]. The $\varepsilon$ value was a spatial frequency $\left(\mathrm{pixel}^{-1}\right.$ ) calculated as the inverse of the distance separating the two points corresponding to $20 \%$ and $80 \%$ of the difference between the minimum and maximum intensities along this line.

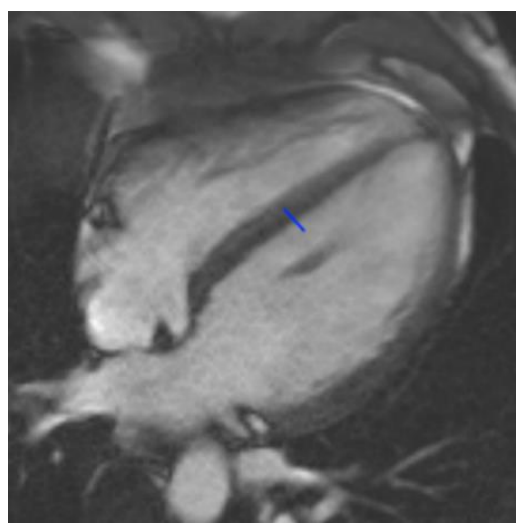

(a)

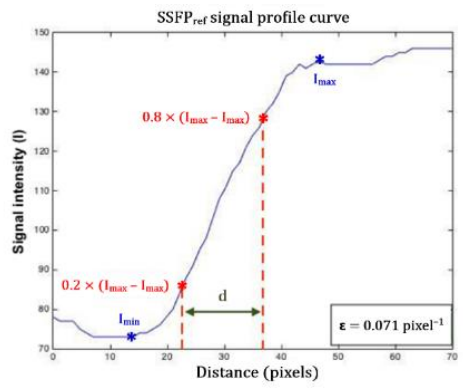

(d)

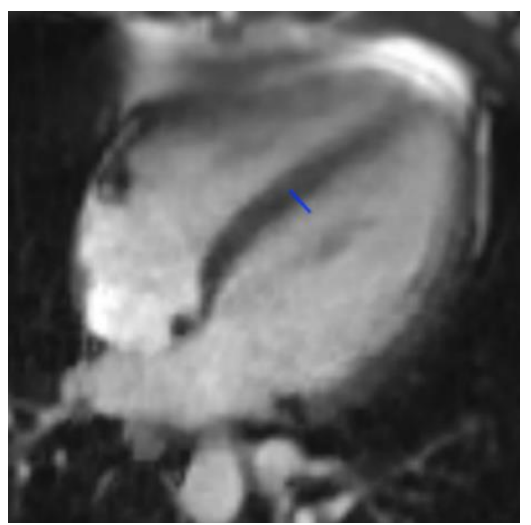

(b)

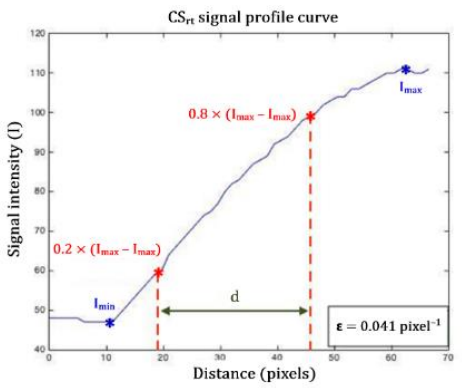

(e)

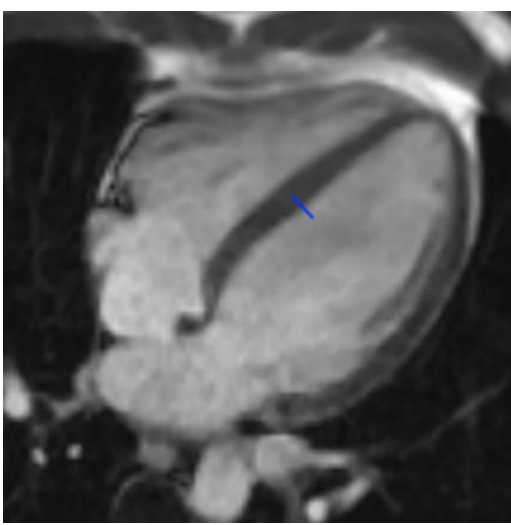

(c)

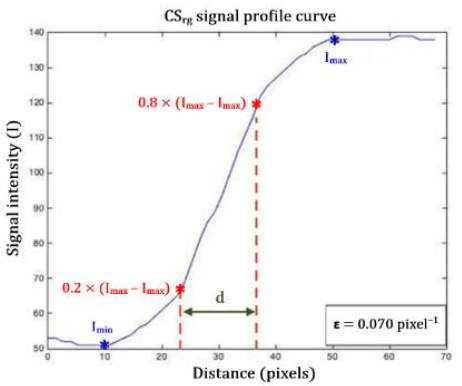

$(\mathbf{f})$

Figure 1. Edge sharpness measurement. An orthogonal profile line is drawn across the border between the interventricular myocardium and the left ventricular blood pool (blue line) on a 4-chamber view at end-diastole provided by (a) the reference segmented SSFP sequence, (b) the real-time compressed sensing sequence and (c) the retrogated compressed sensing prototype, providing intensity profiles (blue curves) along the line for (d) reference, (e) real-time and (f) prototype sequences. The edge sharpness was calculated as the inverse of the distance $d$ (in pixels) from the positions corresponding to $20 \%$ and $80 \%$ (red stars) of the difference between the maximum and minimum signal intensities (black crosses) along the profile line and was expressed in pixel $^{-1}$. Abbreviations: SSFP $_{\text {ref }}$, reference steady-state free precession; $\mathrm{CS}_{\mathrm{rt}}$, real-time compressed sensing; $\mathrm{CS}_{\mathrm{rg}}$, retrogated compressed sensing; $\varepsilon$, edge sharpness; $\mathrm{Ymax}$, maximum signal intensity; Ymin, minimum signal intensity; $d$, distance along the intensity (pixels). 


\subsection{Functional Evaluation}

Assessment of end-diastolic volumes (EDV), end-systolic volumes (ESV), stroke volumes (SV) and EF were performed for both ventricles as well as LV mass (LVM). These parameters were measured on short-axis stacks with semi-automated segmentation with manual correction of the LV endocardium and epicardium while manual segmentation of the RV endocardium was necessary, using dedicated $4 \mathrm{D}$ analysis software (Cardiac MR analysis workflow, Syngo.via VB30A, Siemens Healthcare, Erlangen, Germany). Fourchamber, LV and RV 2-chamber slices were used to define mitral and tricuspid valve planes to ensure optimal assessment of ventricular bases.

\subsection{Conditions of Image Analysis}

The three datasets were independently analyzed by a 4-year experience CMR radiologist (LG). After anonymization and randomization performed for each sequence, each dataset was analyzed separately. Each analysis session, which evaluated all the images of the same sequence, was separated from the previous one by one month. A second radiologist with 11 years of experience in $\mathrm{CMR}(\mathrm{BL})$ segmented both ventricles on $\mathrm{CS}_{\mathrm{rg}}$ images for interrater variability assessment.

\subsection{Statistics Analysis}

Categorical data were presented as numbers (percentage) and continuous variables as mean \pm standard deviation (SD) $(95 \%$ confidence interval (CI)) in the case of normal distribution or median (range: minimum-maximum) in other cases. Variable normality was assessed using the D'Agostino-Pearson test.

Paired Wilcoxon signed-rank test was used to compare subjective image qualities and acquisition qualities between $\mathrm{CS}_{\mathrm{rg}}$ and $\mathrm{SSFP}_{\text {ref }}$ or $\mathrm{CS}_{\mathrm{rt}}$. An analysis of variance was performed to compare edge sharpness and acquisition times of the three sequences. $\mathrm{CS}_{\mathrm{rg}}$ and $\mathrm{SSFP}_{\text {ref }}$ mean functional parameters were compared using a Student's $t$ test, with linear regression and Bland-Altman analysis to assess the agreements between both methods. Inter and intra-observer variabilities were assessed using intra-class coefficient correlation. Significance of the test was defined by values of $p<0.05$.

As for valvular regurgitations and WMD visualization, a receiver operating characteristic (ROC) curve was used. Statistical analysis was performed using dedicated commercially available software (MedCalc 14.8.1.0, MedCalc Software, Ostend, Belgium).

\section{Results}

\subsection{Population Description}

Demographics data are listed in Table 3. The 30 patients ( 22 men, 8 women; mean age: $48.0 \pm 21.0(\mathrm{SD})$ years; $95 \% \mathrm{CI}$ : $40.2-55.9$ years) were referred for: heart valve disease $(n=7 / 30 ; 23.3 \%)$, ischemic cardiopathy $(n=5 / 30 ; 16.7 \%)$; dilated cardiomyopathy $(n=5 / 30 ; 16.7 \%)$, myocarditis $(n=5 / 30 ; 16.7 \%)$, left ventricular hypertrophy $(n=5 / 30$; $16.7 \%)$ and infiltrative cardiomyopathy $(n=3 / 30 ; 10 \%)$. All patients could fully complete $\mathrm{CS}_{\mathrm{rt}}$ and $\mathrm{CS}_{\mathrm{rg}}$ breath-holdings.

Table 3. Study population characteristics.

\begin{tabular}{cccc}
\hline & Mean \pm SD (95\% CI) & Minimum Value & Maximum Value \\
\hline Age-years & $48.0 \pm 21.0(40.2-55.9)$ & 18 & 87 \\
Weight-kg & $73.9 \pm 12.1(69.4-78.5)$ & 53 & 105 \\
Height-cm & $172.5 \pm 8.3(169.4-175.6)$ & 157 & 189 \\
Body surface area-m ${ }^{2}$ & $1.87 \pm 0.17(1.80-1.93)$ & 1.55 & 2.22 \\
Body mass index-kg/m ${ }^{2}$ & $24.8 \pm 3.6(23.5-26.2)$ & 19.8 & 33.7 \\
Heart rate-beats per minute & $73.8 \pm 13.5(68.7-78.9)$ & 54 & 101 \\
\hline
\end{tabular}




\subsection{Scan Time and Image Quality}

SSFP $_{\text {ref }}$ mean scan time was $485.4 \pm 83.3$ (SD) s (95\% CI: 454.3-516.5 s) while CS $_{\text {rt }}$ scan time was $23.9 \pm 5.2$ (SD) s (95\% CI: 21.9-25.8 s) and CS $_{\text {rg }}$ scan time was $58.3 \pm 15.1$ (SD) s (95\% CI: 53.7-64.2 s) $(p<0.0001)$. Compared to SSFP ${ }_{\text {ref }}$, the mean acceleration factor provided by $\mathrm{CS}_{\mathrm{rg}}$ was $8.7 \pm 2.6$ (SD) (95\% CI: 4.5-15.7). A mean number of $15.0 \pm 1.2(\mathrm{SD})$ slices (95\% CI: 14.6-15.4 slices) was acquired with each technique. For $\mathrm{CS}_{\mathrm{rg}}$ reconstruction of the full dataset, the mean time was $82.9 \pm 23.4$ (SD) s (95\% CI: 77.4-110.1 s).

All images were rated as diagnostic. $\mathrm{CS}_{\mathrm{rg}}$ subjective quality score (median: 4 ; range: $2-4)$ was higher than the one provided by $\mathrm{CS}_{\mathrm{rt}}$ (median: 3; range: $\left.2-4 ; p=0.0008\right)$. $\mathrm{SSFP}_{\text {ref }}$ overall quality score (median: 4; range: $2-4)$ was not different from $\mathrm{CS}_{\mathrm{rg}}$ score $(p=0.31)$.

Regarding the acquisition quality based on the EuroCMR registry, the $\mathrm{CS}_{\mathrm{rg}}$ sequence (median: 0; range: 0-3) was not different from either the $\mathrm{SSFP}_{\text {ref }}$ acquisition (median: 0; range: $0-3 ; p=0.38$ ) or the $\mathrm{CS}_{\mathrm{rt}}$ cine (median: 0 ; range: $0-3 ; p=0.83$ ).

The $\mathrm{CS}_{\mathrm{rg}}$ demonstrated a significantly better edge sharpness than $\mathrm{CS}_{\mathrm{rt}}\left(\varepsilon_{\mathrm{CSrg}}=\right.$ $0.083 \pm 0.013$ (SD) pixel $^{-1}$ (95\% CI: 0.078-0.087 pixel $^{-1}$ ) versus $\varepsilon_{\mathrm{CSrt}}=0.070 \pm 0.011$ (SD) pixel $^{-1}$ (95\% CI: 0.066-0.074 pixel $\left.\left.^{-1}\right) ; p=0.0004\right)$. Moreover, no significant difference was demonstrated between $\mathrm{CS}_{\mathrm{rg}}$ and $\mathrm{SSFP}_{\text {ref }}\left(\varepsilon_{\text {SSFPref }}=0.075 \pm 0.016(\mathrm{SD}) \mathrm{pixel}^{-1}\right.$ (95\% CI: 0.069-0.081 pixel $^{-1}$ ); $p=0.0516$ ) (Figure 2; Figure 3; Video S1; Video S2 (Supplementary Materials)).

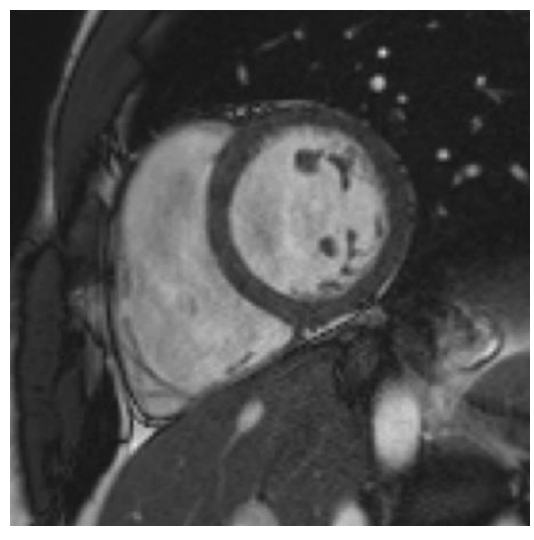

(a)

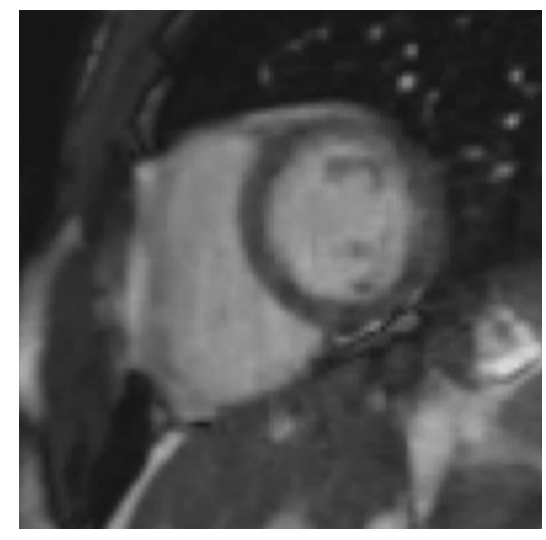

(b)

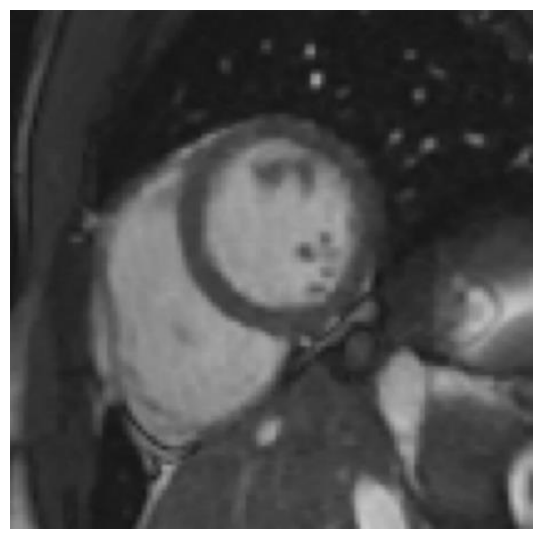

(c)

Figure 2. Midventricular short-axis cine slice acquired with the three cine sequences in a 37-year-old patient referred for myocarditis suspicion. (a) Reference steady-state free precession cine: Likert scale $=4 / 4$; EuroCMR score $=0 / 10$; $\varepsilon=0.082$ pixel $^{-1} ; \mathrm{LVEF}=59 \%, \mathrm{LVEDV}=135 \mathrm{~mL}, \mathrm{LVM}=83 \mathrm{~g}, \mathrm{RVEF}=63 \%$, RVEDV $=159 \mathrm{~mL}$; (b) Real-time compressed sensing cine: Likert scale $=4 / 3$; EuroCMR score $=0 / 10 ; \varepsilon=0.056$ pixel $^{-1}$; LVEF $=60 \%, \mathrm{LVEDV}=133 \mathrm{~mL}, \mathrm{LVM}=81 \mathrm{~g}$, RVEF $=61 \%$, RVEDV = $153 \mathrm{~mL}$; (c) Retrogated compressed sensing cine: Likert scale = 4/4; EuroCMR score = 0/10; $\varepsilon=0.081$ pixel $^{-1} ; \mathrm{LVEF}=58 \%, \mathrm{LVEDV}=133 \mathrm{~mL}, \mathrm{LVM}=86 \mathrm{~g}, \mathrm{RVEF}=62 \%, \mathrm{RVEDV}=164 \mathrm{~mL}$. Abbreviations: $\varepsilon$, edge sharpness; LVEF, left ventricular ejection fraction; LVEDV, left ventricular end-diastolic volume; LVM, left ventricular myocardial mass; RVEF, right ventricular ejection fraction; RVEDV, right ventricular end-diastolic volume. 


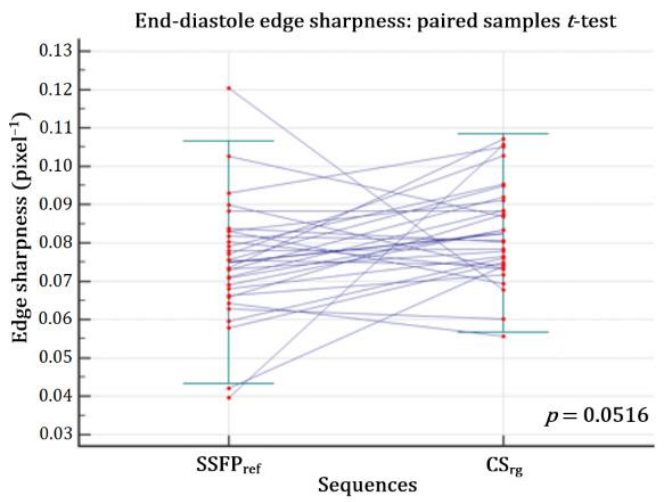

(a)

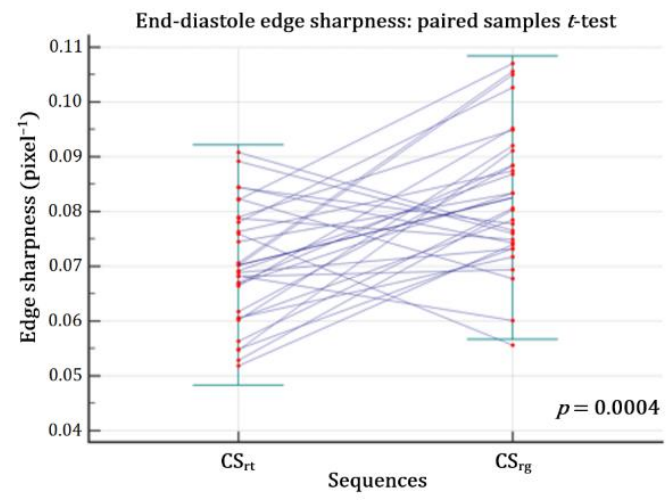

(b)

Figure 3. Edge sharpness comparison assessed at end-diastole. (a) There was no significant difference regarding $\varepsilon$ between $\mathrm{CS}_{\mathrm{rg}}$ and $\mathrm{SSFP}_{\text {ref }}(p=0.0516)(\mathbf{b})$ but $\mathrm{CS}_{\mathrm{rg}}$ significantly improved $\varepsilon$ while compared with $\mathrm{CS}_{\mathrm{rt}}(p=0.0004)$. Abbreviations: $\mathrm{CS}_{\mathrm{rg}}$, retrogated compressed sensing; $\mathrm{CS}_{\mathrm{rt}}$, real-time compressed sensing; $\mathrm{SSFP}_{\text {ref }}$, reference steady-state free precession; $\varepsilon$, edge sharpness.

\subsection{Volumes, Functions and Mass Quantification}

Good agreements were yielded by Bland-Altman and linear regression analyses for both LV (Figure 4) and RV (Figure 5) assessments. No significant difference was demonstrated regarding LVM, LV and RV volumes (EDV, ESV, SV) and EF ( $p>0.05$ ) (Table 4). Intrarater variability was excellent, demonstrating intraclass correlation coefficients (ICC) greater than 0.99 for both ventricles, as were interrater variabilities for LV (ICC $\geq 0.97$ ) and RV (ICC $\geq 0.96)$.

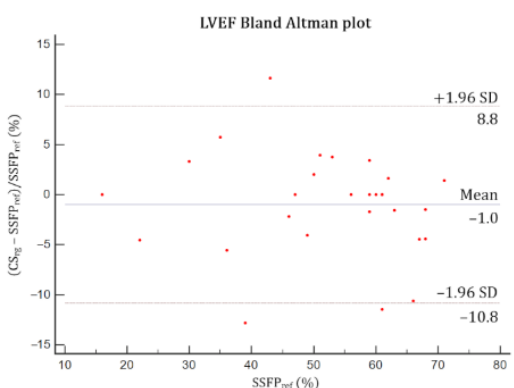

(a)

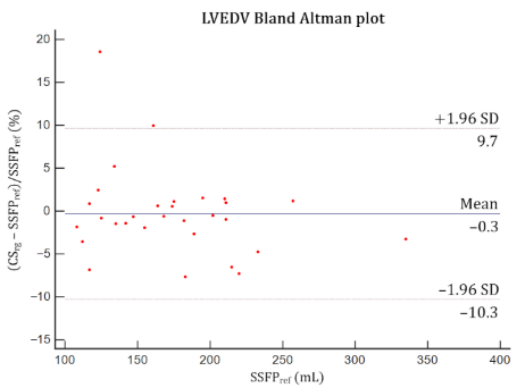

(c)

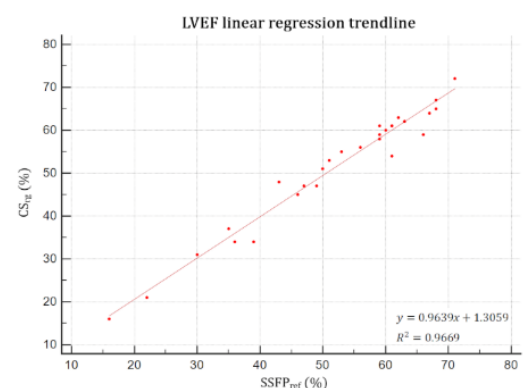

(b)

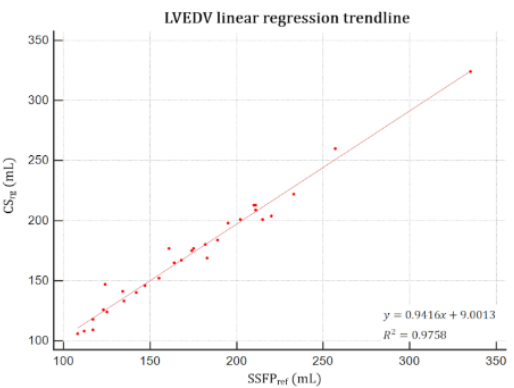

(d)

Figure 4. Cont. 


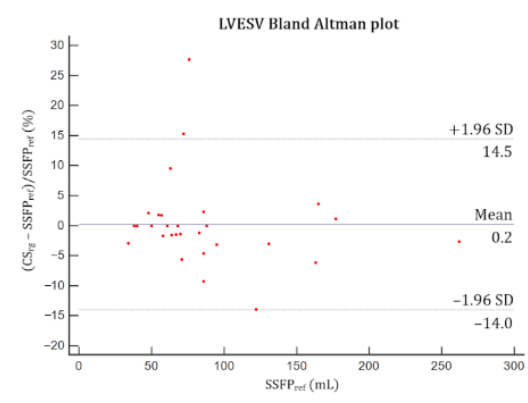

(e)

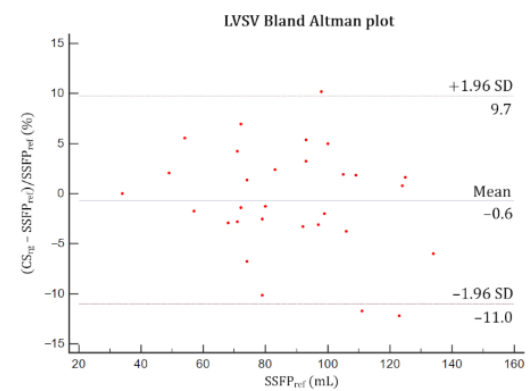

(g)

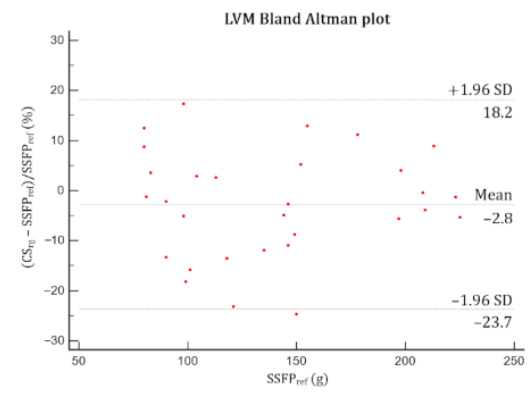

(i)

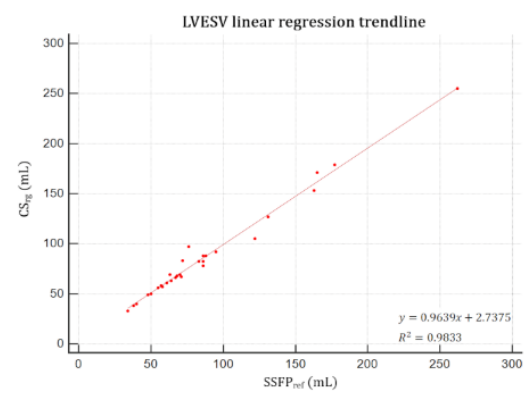

$(\mathbf{f})$

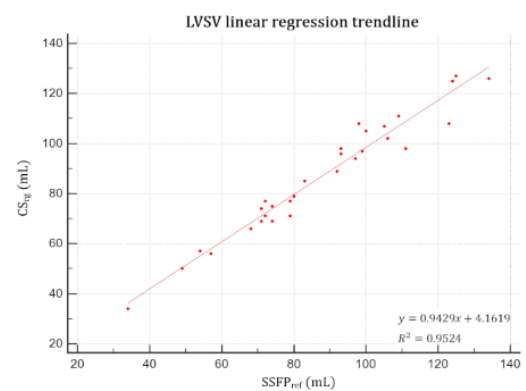

(h)

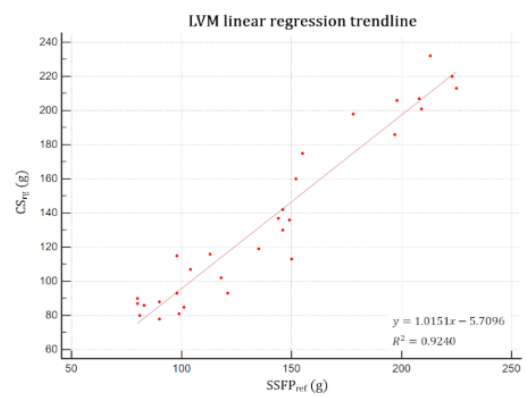

(j)

Figure 4. Bland-Altman plots and linear regression trendlines applied to left ventricular functional parameters quantifications. Left column: Bland-Altman plots for (a) LVEF, (c) LVEDV, (e) LVESV, (g) LVSV and (i) LVM. Solid blue lines are the mean differences between parameters measured with $\mathrm{SSFP}_{\text {ref }}$ and $\mathrm{CS}_{\mathrm{rg}}$ sequences and dashed red lines are the $95 \%$ limits of agreement. Right column: linear regression trend lines for (b) LVEF, (d) LVEDV, (f) LVESV, (h) LVSV and (j) LVM. Abbreviations: $\mathrm{SSFP}_{\text {ref }}$, reference steady-state free precession; $\mathrm{CS}_{\mathrm{rg}}$, retrogated compressed sensing; $\mathrm{SD}$, standard deviation; LVEF, left ventricular ejection fraction; LVEDV, left ventricular end-diastolic volume; LVESV, left ventricular end-systolic volume; LVSV, left ventricular stroke volume; LVM, left ventricular mass. 


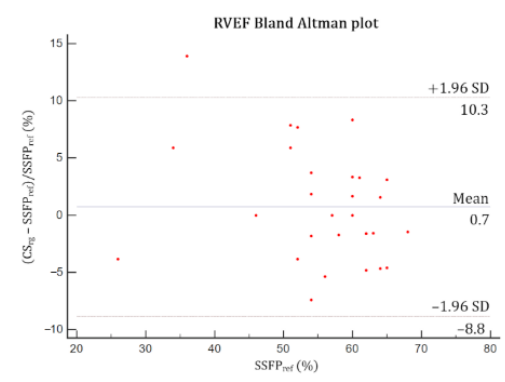

(a)

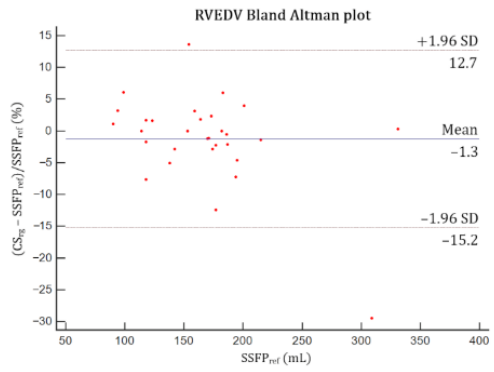

(c)

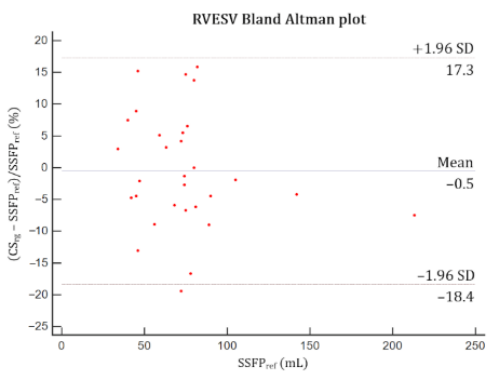

(e)

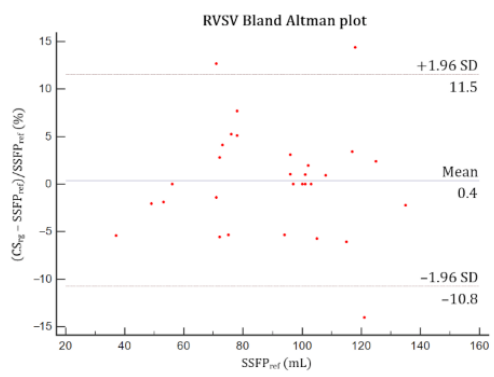

(g)

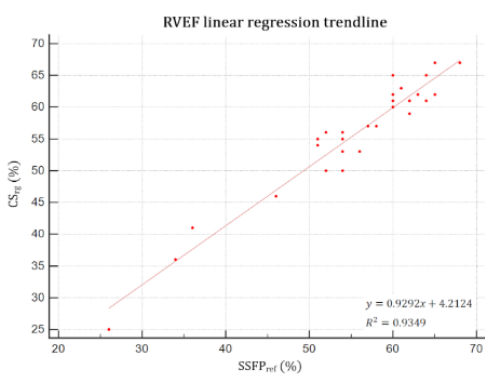

(b)

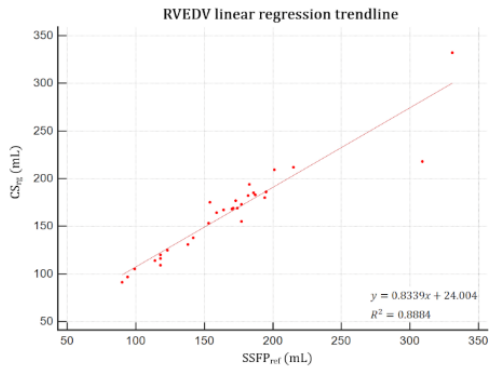

(d)

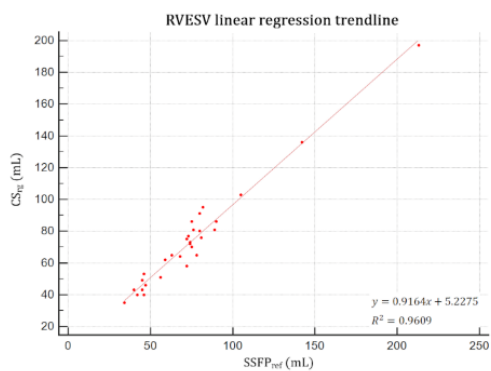

(f)

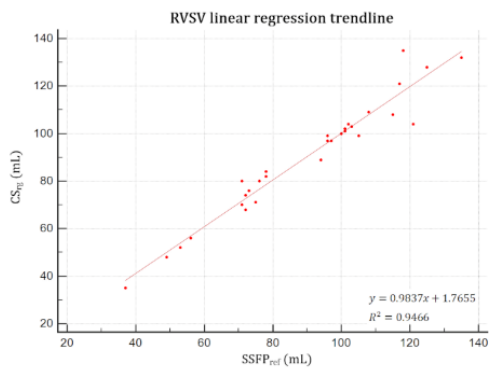

(h)

Figure 5. Bland-Altman plots and linear regression trendlines applied to right ventricular functional parameters quantifications. Left column: Bland-Altman plots for (a) RVEF, (c) RVEDV, (e) RVESV, and (g) RVSV. Solid blue lines are the mean differences between parameters measured with SSFP ref and $\mathrm{CS}_{\mathrm{rg}}$ sequences and dashed red lines are the 95\% limits of agreement. Right column: linear regression trend lines for (b) RVEF, (d) RVEDV, (f) RVESV, and (h) RVSV. Abbreviations: SSFP ref, reference steady-state free precession; $\mathrm{CS}_{\mathrm{rg}}$, retrogated compressed sensing; $\mathrm{SD}$, standard deviation; RVEF, right ventricular ejection fraction; RVEDV, right ventricular end-diastolic volume; RVESV, right ventricular end-systolic volume; RVSV, right ventricular stroke volume. 
Table 4. Functional parameters segmented on both the reference steady-state free-precession and retrogated compressedsensing cine.

\begin{tabular}{|c|c|c|c|c|c|c|}
\hline & \multirow{2}{*}{$\begin{array}{c}\text { SSFP }_{\text {ref }} \text { Sequence } \\
(\text { Mean } \pm \text { SD }(95 \% \text { CI }))\end{array}$} & \multirow{2}{*}{$\begin{array}{c}\mathrm{CS}_{\mathrm{rg}} \text { Sequence } \\
(\text { Mean } \pm \operatorname{SD}(95 \% \mathrm{CI}))\end{array}$} & \multirow{2}{*}{$\begin{array}{c}\text { Difference } \\
(\text { Mean } \pm \text { SD }(95 \% \text { CI) })\end{array}$} & \multirow{2}{*}{ Paired $t$ Test $p$} & \multicolumn{2}{|c|}{ ICC } \\
\hline & & & & & Inter & Intra \\
\hline LVEF-\% & $\begin{array}{l}52.7 \pm 14.1 \\
(47.5-58.0)\end{array}$ & $\begin{array}{l}52.1 \pm 13.8 \\
(47.0-57.3)\end{array}$ & $\begin{array}{c}-0.6 \pm 2.6 \\
(-1.6 \text { to }-0.4)\end{array}$ & 0.21 & 0.99 & 0.9996 \\
\hline LVEDV-mL & $\begin{array}{c}174.1 \pm 50.3 \\
(155.3-192.9)\end{array}$ & $\begin{array}{c}173.0 \pm 48.0 \\
(155.1-190.9)\end{array}$ & $\begin{array}{c}-1.2 \pm 8.0 \\
(-4.2 \text { to }-1.8)\end{array}$ & 0.43 & 0.99 & 0.9994 \\
\hline LVESV—mL & $\begin{array}{l}86.8 \pm 49.4 \\
(68.4-105.2)\end{array}$ & $\begin{array}{l}86.4 \pm 48.0 \\
(68.5-104.3)\end{array}$ & $\begin{array}{c}-0.4 \pm 6.5 \\
(-2.8 \text { to }-2.0)\end{array}$ & 0.74 & 0.98 & 0.9906 \\
\hline LVSV—mL & $\begin{array}{l}87.5 \pm 24.0 \\
(78.6-96.5)\end{array}$ & $\begin{array}{l}86.7 \pm 23.2 \\
(78.0-95.4)\end{array}$ & $\begin{array}{c}-0.8 \pm 5.3 \\
(-2.8 \text { to }-1.1)\end{array}$ & 0.39 & 0.98 & 0.9932 \\
\hline $\mathrm{LVM}-\mathrm{g}$ & $\begin{array}{l}139.5 \pm 47.7 \\
(121.6-157.3)\end{array}$ & $\begin{array}{l}135.9 \pm 50.4 \\
(117.0-154.7)\end{array}$ & $\begin{array}{l}-3.6 \pm 13.9 \\
(-8.8 \text { to } 1.6)\end{array}$ & 0.17 & 0.97 & 0.9994 \\
\hline RVEF-\% & $\begin{array}{l}55.8 \pm 9.7 \\
(52.2-59.4)\end{array}$ & $\begin{array}{l}56.0 \pm 9.3 \\
(52.6-59.5)\end{array}$ & $\begin{array}{c}0.3 \pm 2.4 \\
(-0.7 \text { to } 1.2)\end{array}$ & 0.56 & 0.96 & 0.9965 \\
\hline RVEDV—mL & $\begin{array}{c}167.0 \pm 53.6 \\
(147.0-187.0)\end{array}$ & $\begin{array}{c}163.2 \pm 47.4 \\
(145.5-180.9)\end{array}$ & $\begin{array}{c}-3.8 \pm 18.2 \\
(-10.5 \text { to } 3.05)\end{array}$ & 0.27 & 0.98 & 0.9968 \\
\hline $\mathrm{RVESV}-\mathrm{mL}$ & $\begin{array}{l}74.1 \pm 34.3 \\
(61.3-86.9)\end{array}$ & $\begin{array}{l}73.1 \pm 32.0 \\
(61.1-85.0)\end{array}$ & $\begin{array}{l}-1.0 \pm 6.9 \\
(-3.6 \text { to } 1.6)\end{array}$ & 0.45 & 0.96 & 0.9934 \\
\hline RVSV—mL & $\begin{array}{l}89.8 \pm 24.3 \\
(80.8-98.9)\end{array}$ & $\begin{array}{l}90.1 \pm 24.5 \\
(81.0-99.3)\end{array}$ & $\begin{array}{c}-0.3 \pm 5.7 \\
(-1.8 \text { to } 2.4)\end{array}$ & 0.77 & 0.97 & 0.9961 \\
\hline
\end{tabular}

ICC assessed the inter and intrarater agreements for ventricular segmentations. The significance of Student's $t$ test is defined by values of $p<0.05$ Abbreviations: $\mathrm{SSFP}_{\text {ref }}$, reference steady-state free-precession cine; $\mathrm{CS}_{\mathrm{rg}}$, retrogated compressed-sensing cine; $\mathrm{SD}$, standard deviation; $95 \% \mathrm{CI}, 95 \%$ confidence interval; LV, left ventricular; RV, right ventricular; EF, ejection fraction; EDV, end-diastolic volume; ESV, end-systolic volume; SV, stroke volume; LVM, left ventricular mass; ICC, intraclass correlation coefficient; Inter, interrater; Intra, intrarater.

\section{Discussion}

Our clinical study performed a comprehensive evaluation of a retrogated CS sequence in daily practice. Results are in line with preliminary $\mathrm{CS}_{\mathrm{rg}}$ tests on eight healthy volunteers reported by Forman et al., regarding LVEF, LVEDV and LVESV, which were similar to SSFP $_{\text {ref }}$ quantification [34]. However, the study population was small and LVM, RV volumes and EF were not assessed. In the present study, no significant difference was demonstrated regarding LVM, LV and RV volumes (EDV, ESV and SV) and EF.

First-generation 49-ms temporal resolution $\mathrm{CS}_{\text {rt }}$ sequence provided LVEDV underestimation and LVM overestimation, which were clinically insignificant or smaller than intra or interrater variabilities [17,22,25,26,35]. Moreover, LVEDV underestimation was also reported with other acceleration techniques such as radial gradient-echo or k-space parallel imaging [36,37]. These differences were not depicted with $\mathrm{CS}_{\mathrm{rg}}$. This might be explained by the improved edge sharpness facilitating segmentation, retrospective ECG gating allowing acquisition of the last phases of the cardiac cycle and the better temporal resolution (37 $\mathrm{ms}$ ) [38]. This observation is in line with previous studies suggesting an optimal temporal resolution for accurate steady-state free precession quantification below $45 \mathrm{~ms}$ [29]. Since quantification is a major CMR point of interest, the absence of significant difference regarding each LV and RV parameter seems promising for clinical implementation [1]. Moreover, the high intra and interrater reproducibility allows the use of $\mathrm{CS}_{\mathrm{rg}}$ for chronic status follow-up such as heart failure, anthracycline induced cardiotoxicity and other cardiomyopathies [39-41].

The overall image quality alteration using $\mathrm{CS}_{\mathrm{rt}}$ has already been assessed in a previous publication [22]. The higher score provided by $\mathrm{CS}_{\mathrm{rg}}$ confirmed the image quality improvement. Indeed, $\mathrm{CS}_{\mathrm{rt}}$ interpolation was responsible for smoothed images, which required physicians to get used to this rendering. The absence of difference between acquisitions qualities (EuroCMR quality score) was expected. Indeed, no difference had been demonstrated regarding the first-generation CS sequence. The evaluated prototype features partial Fourier switch-off and segmented acquisition, which were not supposed to generate more artifacts. Regarding edge sharpness, the absence of interpolation and 
acquisition of more data improves boarder delineation. Not only was $\varepsilon_{\mathrm{CS}}$ better than $\varepsilon_{\text {CSrg }}$ but it was similar to $\varepsilon_{\text {SSFPref. }}$. Edge sharpness was chosen as a metric for intrinsic image quality assessment due to its simple and reproducible implementation. Moreover, edge sharpness is an estimate of the edge spread function whose derivative is a line-spread function [33]. Fast Fourier transform of the latter gives the task-based modulation transfer function $\left(\mathrm{MTF}_{\text {Task }}\right)$; edge sharpness can be considered as a reasonable approximation of $\mathrm{MTF}_{\text {Task}}$, which is widely used to evaluate the spatial frequency response of an imaging system, even using iterative reconstructions [42].

Predictably, $\mathrm{CS}_{\mathrm{rg}}$ scan time was approximatively twice as long as the first-generation CS sequence since acquisitions were segmented on two heart beats in contrast to real-time imaging. However, as compared to SSFP ref, the mean CS $_{\text {rg }}$ scan time of about $60 \mathrm{~s}$ still provides an 8.7-fold acceleration factor. The study protocol divided $\mathrm{CS}_{\mathrm{rg}}$ acquisition in 3 stacks to shorten breath-holdings. Nevertheless, this setting is adjustable, such as the number of heart beats required for one slice to reduce apneas or increase spatiotemporal resolution. We chose to use CS to improve scan time, with workflow fluency being a major point of concern. However, it is possible to take advantage of this acceleration to improve spatial resolution, or maybe more interestingly, temporal resolution. Indeed, in the field of feature tracking in CMR, a resolution at least over 30 frames per cycle is recommended for accurate strain assessment, which is more time-consuming when using $\mathrm{SSFP}_{\text {ref }}$ [43]. Such settings may facilitate further feature tracking studies, though the impact of CS based reconstructions should be evaluated regarding strain analysis reliability.

The image quality improvement provided by the prototype $\mathrm{CS}_{\mathrm{rg}}$ as compared to the first-generation real-time $\mathrm{CS}_{\mathrm{rt}}$ should facilitate the spread of $\mathrm{CS}$ use in daily practice. However, the 2-shot acquisition is responsible for the loss of the real-time acquisition. Futures generations of CS cine sequences, maintaining the $\mathrm{CS}_{\mathrm{rt}}$ real-time feature and the $\mathrm{CS}_{\mathrm{rg}}$ image quality, should be oriented towards the application of additional motion correction algorithms to provide free-breathing acquisition with preserved image quality.

\section{Limitations}

The size of the population was limited and cannot represent the whole variety of cardiac conditions encountered in daily practice. Nevertheless, the main objective of this study was to assess the image quality recovery as compared to $\mathrm{SSFP}_{\text {ref }}$ and $\mathrm{CS}_{\mathrm{rt}}$. To facilitate the standardized assessment of edge sharpness, we chose to exclude congenital heart disease anatomy. Among the cardiac conditions assessed by CMR, dilated cardiomyopathy is a frequent pathology, usually responsible for shortness of breath, in which the LV wall may be thinned. The decreased wall thickness may be challenging for endocardium and epicardium delineation, thus impairing LV mass assessment. The impact of LV wall thickness for this assessment, for instance in dilated cardiomyopathy, could not be evaluated because of an insufficient subgroup. Nevertheless, we assume that the edge sharpness provided by $\mathrm{CS}_{\mathrm{rg}}$ should help to distinguish the endocardium from the epicardium as compared to the first-generation $\mathrm{CS}_{\mathrm{rt}}$ cine that provided blurrier borders. Although a high acceleration factor was demonstrated using $\mathrm{CS}_{\mathrm{rg}}$, the improvement of $\mathrm{CMR}$ tolerance can only be assumed since this parameter was not evaluated. However, we suppose that the lower number of breath-holdings required by $\mathrm{CS}_{\mathrm{rg}}$ should help dyspneic or claustrophobic patients undergo CMR examinations. This acceleration might be overestimated and must be interpreted cautiously since the two $1.6 \times 1.6 \mathrm{~mm}^{2}$ in-plane resolution CS sequences were compared with a $1.3 \times 1.3 \mathrm{~mm}^{2}$ in-plane resolution reference sequence. However, Miller et al. demonstrated that the maximal accuracy for functional parameters quantification using SSFP sequence was reached between 1 and 2-mm in-plane spatial resolution [29]. Even though further acquisitions could be performed during $\mathrm{CS}_{\mathrm{rg}}$ reconstruction during this study (SSFP ref images were acquired first and available), up to 2 min were necessary for images to be reconstructed and displayed to set the next sequences orientation in case of exclusive use of $\mathrm{CS}_{\mathrm{rg}}$. Wall motion abnormalities were not evaluated, but the increased temporal resolution provided by segmented acquisition and the absence of interpolation 
should not impair their visualization on $\mathrm{CS}_{\mathrm{rg}}$ since the first generation of compressed sensing cine was demonstrated to be reliable for this evaluation (Video S3 (Supplementary Materials)) [44]. The heart rate of the evaluated patients ranged from 54 to 101 beats per minute (R-R intervals: $594 \mathrm{~ms}$ to $1111 \mathrm{~ms}$ ). Since $\mathrm{CS}_{\mathrm{rg}}$ is a 2-shot sequence and its temporal resolution is constant $(37 \mathrm{~ms})$, the amount of data acquired during the acquisition must vary and may have an impact on the quality of the reconstructed cine images and consequently on functional assessment. The impact of heart rate on image quality was not evaluated in this study due to the limited size of the study population. Finally, only sinus rhythm patients were enrolled in this study. Even though a real-time acquisition is more robust than a segmented acquisition versus arrhythmia, the need of only two heart beats per slice should be more arrhythmia-proof than the conventional 8-heart-beat $\mathrm{SSFP}_{\text {ref }}$ Further comparison with other acceleration techniques such as generalized autocalibrating partial parallel acquisition would be interesting since the later requires more heart beats for identical temporal and spatial resolutions [45].

\section{Conclusions}

$\mathrm{CS}_{\text {rg }}$ allows reliable quantification of $\mathrm{LV}$ and RV volumes, EF and mass providing similar objective and subjective image quality to SSFP $_{\text {ref. }}$. Performed in clinical conditions, $\mathrm{CS}_{\mathrm{rg}}$ is promising in terms of workflow improvement and image quality recovering in comparison with the first-generation real-time $\mathrm{CS}_{\mathrm{rt}}$.

Supplementary Materials: The following are available online at https://www.mdpi.com/article/ 10.3390/jcm10112417/s1, Video S1: Short-axis cine stacks in a 45-year-old woman referred for myocarditis follow-up; Video S2: Four-chamber cine slice in the same patient as Video S1; Video S3, short-axis mid-cavity cine slice in a 68-year-old patient referred for viability assessment after myocardial infarction, demonstrating inferolateral akinesia.

Author Contributions: B.L.: study conception and design, data collection, interpretation and analysis, drafting of the manuscript, critical revision for important intellectual content; C.V.G.: data collection and interpretation, critical revision for important intellectual content; A.C.: study conception, critical revision for important intellectual content; L.G.: data collection, interpretation and analysis, drafting of the manuscript; V.S.: data collection and interpretation, critical revision for important intellectual content; J.P.: data collection and interpretation, critical revision for important intellectual content; A.S.: data collection and interpretation, critical revision for important intellectual content; J.H.: data collection and interpretation, critical revision for important intellectual content; M.S.: study conception and design, critical revision for important intellectual content; C.F.: study conception and design, critical revision for important intellectual content; S.T.: study conception and design, critical revision for important intellectual content; D.M.: study conception, critical revision for important intellectual content; F.P.: study conception and design, data collection, interpretation and analysis, drafting of the manuscript, critical revision for important intellectual content. All authors have read and agreed to the published version of the manuscript.

Funding: This research received no external funding.

Institutional Review Board Statement: The study was approved by the research ethics committee of Lille University Hospital.

Informed Consent Statement: Informed consent was obtained from all subjects involved in the study.

Data Availability Statement: The data presented in this study are available on reasonable request from the corresponding author, subject to approval by the research ethics committee of Lille University Hospital.

Conflicts of Interest: B.L.; C.V.G; A.C.; L.G.; V.S.; J.P.; A.S.; J.H.; D.M.; F.P. have no competing interest. They are employed by an institution engaged in a contractual collaboration with Siemens Healthcare. M.S.; C.F.; S.T. are employees of Siemens Healthcare GmbH. 


\section{References}

1. Pennell, D.J.; Sechtem, U.P.; Higgins, C.B.; Manning, W.J.; Pohost, G.M.; Rademakers, F.E.; van Rossum, A.C.; Shaw, L.J.; Yucel, E.K.; Society for Cardiovascular Magnetic Resonance; et al. Clinical indications for cardiovascular magnetic resonance (CMR): Consensus panel report. Eur. Heart J. 2004, 25, 1940-1965. [CrossRef]

2. Maceira, A.M.; Prasad, S.K.; Khan, M.; Pennell, D.J. Normalized left ventricular systolic and diastolic function by steady state free precession cardiovascular magnetic resonance. J. Cardiovasc. Magn. Reson. 2006, 8, 417-426. [CrossRef]

3. Maceira, A.M.; Prasad, S.K.; Khan, M.; Pennell, D.J. Reference right ventricular systolic and diastolic function normalized to age, gender and body surface area from steady-state free precession cardiovascular magnetic resonance. Eur. Heart J. 2006, 27, 2879-2888. [CrossRef]

4. Curtis, J.P.; Sokol, S.I.; Wang, Y.; Rathore, S.S.; Ko, D.T.; Jadbabaie, F.; Portnay, E.L.; Marshalko, S.J.; Radford, M.J.; Krumholz, H.M. The association of left ventricular ejection fraction, mortality, and cause of death in stable outpatients with heart failure. J. Am. Coll. Cardiol. 2003, 42, 736-742. [CrossRef]

5. Karamitsos, T.D.; Francis, J.M.; Myerson, S.; Selvanayagam, J.B.; Neubauer, S. The role of cardiovascular magnetic resonance imaging in heart failure. J. Am. Coll. Cardiol. 2009, 54, 1407-1424. [CrossRef]

6. Knauth, A.L.; Gauvreau, K.; Powell, A.J.; Landzberg, M.J.; Walsh, E.P.; Lock, J.E.; del Nido, P.J.; Geva, T. Ventricular size and function assessed by cardiac MRI predict major adverse clinical outcomes late after tetralogy of Fallot repair. Heart Br. Card. Soc. 2008, 94, 211-216. [CrossRef]

7. Kramer, C.M.; Barkhausen, J.; Flamm, S.D.; Kim, R.J.; Nagel, E. Society for Cardiovascular Magnetic Resonance Board of trustees task force on standardized protocols standardized cardiovascular magnetic resonance (CMR) protocols 2013 update. J. Cardiovasc. Magn. Reson. 2013, 15, 91. [CrossRef]

8. Vincenti, G.; Monney, P.; Chaptinel, J.; Rutz, T.; Coppo, S.; Zenge, M.O.; Schmidt, M.; Nadar, M.S.; Piccini, D.; Chèvre, P.; et al. Compressed sensing single-breath-hold CMR for fast quantification of lv function, volumes, and mass. J. Am. Coll. Cardiol. Imaging 2014, 7, 882-892. [CrossRef]

9. Candès, E.J.; Romberg, J.K.; Tao, T. Stable signal recovery from incomplete and inaccurate measurements. Commun. Pure Appl. Math. 2006, 59, 1207-1223. [CrossRef]

10. Donoho, D.L. Compressed Sensing. IEEE Trans. Inf. Theory 2006, 52, 1289-1306. [CrossRef]

11. Lustig, M.; Donoho, D.; Pauly, J.M. Sparse MRI: The application of compressed sensing for rapid MR imaging. Magn. Reson. Med. 2007, 58, 1182-1195. [CrossRef]

12. Lustig, M.; Santos, J.M.; Donoho, D.L.; Pauly, J.M. K-t SPARSE: High frame rate dynamic MRI exploiting spatio-temporal sparsity. In Proceedings of the 14th International Society for Magnetic Resonance in Medecine annual meeting, Seattle, WA, USA, 6-12 May 2006.

13. Feng, L.; Srichai, M.B.; Lim, R.P.; Harrison, A.; King, W.; Adluru, G.; Dibella, E.V.R.; Sodickson, D.K.; Otazo, R.; Kim, D. Highly Accelerated real-time cardiac cine MRI using k-t SPARSE-SENSE. Magn. Reson. Med. 2013, 70, 64-74. [CrossRef]

14. Jahnke, C.; Nagel, E.; Gebker, R.; Bornstedt, A.; Schnackenburg, B.; Kozerke, S.; Fleck, E.; Paetsch, I. Four-dimensional single breathhold magnetic resonance imaging using kt-BLAST enables reliable assessment of left- and right-ventricular volumes and mass. J. Magn. Reson. Imaging 2007, 25, 737-742. [CrossRef] [PubMed]

15. Klinke, V.; Muzzarelli, S.; Lauriers, N.; Locca, D.; Vincenti, G.; Monney, P.; Lu, C.; Nothnagel, D.; Pilz, G.; Lombardi, M.; et al. Quality assessment of cardiovascular magnetic resonance in the setting of the European CMR Registry: Description and validation of standardized criteria. J. Cardiovasc. Magn. Reson. 2013, 15, 55. [CrossRef]

16. Bogachkov, A.; Ayache, J.B.; Allen, B.D.; Murphy, I.; Carr, M.L.; Spottiswoode, B.; Schmidt, M.; Zenge, M.O.; Nadar, M.S.; Zuehlsdorff, S.; et al. Right ventricular assessment at cardiac MRI: Initial clinical experience utilizing an IS-SENSE reconstruction. Int. J. Cardiovasc. Imaging 2016, 32, 1081-1091. [CrossRef]

17. Goebel, J.; Nensa, F.; Bomas, B.; Schemuth, H.P.; Maderwald, S.; Gratz, M.; Quick, H.H.; Schlosser, T.; Nassenstein, K. Real-Time SPARSE-SENSE Cardiac Cine MR Imaging: Optimization of image reconstruction and sequence validation. Eur. Radiol. 2016, 26, 4482-4489. [CrossRef]

18. Goebel, J.; Nensa, F.; Schemuth, H.P.; Maderwald, S.; Gratz, M.; Quick, H.H.; Schlosser, T.; Nassenstein, K. Compressed sensing cine imaging with high spatial or high temporal resolution for analysis of left ventricular function. J. Magn. Reson. Imaging 2016 44, 366-374. [CrossRef]

19. Kido, T.; Kido, T.; Nakamura, M.; Watanabe, K.; Schmidt, M.; Forman, C.; Mochizuki, T. Compressed sensing real-time cine cardiovascular magnetic resonance: Accurate assessment of left ventricular function in a single-breath-hold. J. Cardiovasc. Magn. Reson. 2016, 18, 50-60. [CrossRef]

20. Kido, T.; Kido, T.; Nakamura, M.; Watanabe, K.; Schmidt, M.; Forman, C.; Mochizuki, T. Assessment of left ventricular function and mass on free-breathing compressed sensing real-time cine imaging. Circ. J. 2017, 81, 1463-1468. [CrossRef] [PubMed]

21. Lin, A.C.W.; Strugnell, W.; Riley, R.; Schmitt, B.; Zenge, M.; Schmidt, M.; Morris, N.R.; Hamilton-Craig, C. Higher resolution cine imaging with compressed sensing for accelerated clinical left ventricular evaluation. J. Magn. Reson. Imaging 2017, 45, 1693-1699. [CrossRef]

22. Vermersch, M.; Longère, B.; Coisne, A.; Schmidt, M.; Forman, C.; Monnet, A.; Pagniez, J.; Silvestri, V.; Simeone, A.; Montaigne, D.; et al. Compressed sensing real-time cine imaging for assessment of ventricular function, volumes and mass in clinical practice. Eur. Radiol. 2020, 30, 609-619. [CrossRef] 
23. Haubenreisser, H.; Henzler, T.; Budjan, J.; Sudarski, S.; Zenge, M.O.; Schmidt, M.; Nadar, M.S.; Borggrefe, M.; Schoenberg, S.O.; Papavassiliu, T. Right ventricular imaging in 25 seconds: Evaluating the use of sparse sampling cine with iterative reconstruction for volumetric analysis of the right ventricle. Investig. Radiol. 2016, 51, 379-386. [CrossRef]

24. Camargo, G.C.; Erthal, F.; Sabioni, L.; Penna, F.; Strecker, R.; Schmidt, M.; Zenge, M.O.; de Lima, R.S.L.; Gottlieb, I. Realtime cardiac magnetic resonance cine imaging with sparse sampling and iterative reconstruction for left-ventricular measures: Comparison with gold-standard segmented steady-state free precession. Magn. Reson. Imaging 2017, 38, 138-144. [CrossRef]

25. Sudarski, S.; Henzler, T.; Haubenreisser, H.; Dösch, C.; Zenge, M.O.; Schmidt, M.; Nadar, M.S.; Borggrefe, M.; Schoenberg, S.O.; Papavassiliu, T. Free-breathing sparse sampling cine $\mathrm{mr}$ imaging with iterative reconstruction for the assessment of left ventricular function and mass at 3.0 T. Radiology 2017, 282, 74-83. [CrossRef]

26. Vincenti, G.; Piccini, D.; Monney, P.; Chaptinel, J.; Rutz, T.; Coppo, S.; Zenge, M.O.; Schmidt, M.; Nadar, M.S.; Wang, Q.; et al. Preliminary experiences with compressed sensing multislice cine acquisitions for the assessment of left ventricular function: CV_sparse WIP. Magn. Flash 2013, 55, 26-34.

27. Goebel, J.; Nensa, F.; Schemuth, H.P.; Maderwald, S.; Quick, H.H.; Schlosser, T.; Nassenstein, K. Real-time SPARSE-SENSE cine MR imaging in atrial fibrillation: A feasibility study. Acta Radiol. 2017, 58, 922-928. [CrossRef]

28. Weiger, M.; Pruessmann, K.P.; Boesiger, P. Cardiac real-time imaging using SENSE. SENSitivity Encoding Scheme. Magn. Reson. Med. 2000, 43, 177-184. [CrossRef]

29. Miller, S.; Simonetti, O.P.; Carr, J.; Kramer, U.; Finn, J.P. MR imaging of the heart with cine true fast imaging with steady-state precession: Influence of spatial and temporal resolutions on left ventricular functional parameters. Radiology 2002, 223, 263-269. [CrossRef]

30. Roussakis, A.; Baras, P.; Seimenis, I.; Andreou, J.; Danias, P.G. Relationship of number of phases per cardiac cycle and accuracy of measurement of left ventricular volumes, ejection fraction, and mass. J. Cardiovasc. Magn. Reson. 2004, 6, 837-844. [CrossRef]

31. Wetzl, J.; Schmidt, M.; Pontana, F.; Longère, B.; Lugauer, F.; Maier, A.; Hornegger, J.; Forman, C. Single-breath-hold 3-D CINE imaging of the left ventricle using cartesian sampling. Magma 2017, 31, 19-31. [CrossRef]

32. Larson, A.C.; Kellman, P.; Arai, A.; Hirsch, G.A.; McVeigh, E.; Li, D.; Simonetti, O.P. Preliminary investigation of respiratory self-gating for free-breathing segmented cine MRI. Magn. Reson. Med. 2005, 53, 159-168. [CrossRef]

33. Richard, S.; Husarik, D.B.; Yadava, G.; Murphy, S.N.; Samei, E. Towards Task-based assessment of CT performance: System and object MTF across different reconstruction algorithms. Med. Phys. 2012, 39, 4115-4122. [CrossRef] [PubMed]

34. Forman, C.; Kroeker, R.; Schmidt, M. Accelerated 2D cine MRI featuring compressed sensing and ECG-triggered retro-gating. In Proceedings of the 25th International Society for Magnetic Resonance in Medicine annual meeting, Honolulu, HI, USA, 22-27 April 2017.

35. Semelka, R.C.; Tomei, E.; Wagner, S.; Mayo, J.; Kondo, C.; Suzuki, J.; Caputo, G.R.; Higgins, C.B. Normal left ventricular dimensions and function: Interstudy reproducibility of measurements with cine MR imaging. Radiology 1990, 174, 763-768. [CrossRef] [PubMed]

36. Voit, D.; Zhang, S.; Unterberg-Buchwald, C.; Sohns, J.M.; Lotz, J.; Frahm, J. Real-time cardiovascular magnetic resonance at 1.5 T using balanced SSFP and 40 ms resolution. J. Cardiovasc. Magn. Reson. 2013, 15, 79-86. [CrossRef] [PubMed]

37. Eberle, H.C.; Nassenstein, K.; Jensen, C.J.; Schlosser, T.; Sabin, G.V.; Naber, C.K.; Bruder, O. Rapid MR assessment of left ventricular systolic function after acute myocardial infarction using single breath-hold cine imaging with the temporal parallel acquisition technique (TPAT) and 4D guide-point modelling analysis of left ventricular function. Eur. Radiol. 2010, 20, 73-80. [CrossRef]

38. Nacif, M.S.; Zavodni, A.; Kawel, N.; Choi, E.-Y.; Lima, J.A.C.; Bluemke, D.A. Cardiac magnetic resonance imaging and its electrocardiographs (ECG): Tips and tricks. Int. J. Cardiovasc. Imaging 2012, 28, 1465-1475. [CrossRef]

39. Kotwinski, P.; Smith, G.; Sanders, J.; Cooper, J.; Kotwinski, D.; Teis, A.; Mythen, M.; Monty, G.; Jones, A.; Montgomery, H.E.; et al. CMR shows that anthracycline cardiotoxicity is common in women treated for early breast cancer and associated with undiagnosed hypertension; but cannot be reliably detected using late-gadolinium enhancement imaging. J. Cardiovasc. Magn. Reson. 2013, 15, 276. [CrossRef]

40. Patel, A.R.; Kramer, C.M. Role of cardiac magnetic resonance in the diagnosis and prognosis of nonischemic cardiomyopathy. J. Am. Coll. Cardiol. Imaging 2017, 10, 1180-1193. [CrossRef]

41. Ponikowski, P.; Voors, A.A.; Anker, S.D.; Bueno, H.; Cleland, J.G.F.; Coats, A.J.S.; Falk, V.; González-Juanatey, J.R.; Harjola, V.-P.; Jankowska, E.A.; et al. 2016 ESC Guidelines for the diagnosis and treatment of acute and chronic heart failure: The task force for the diagnosis and treatment of acute and chronic heart failure of the European Society of Cardiology (ESC). Developed with the special contribution of the Heart Failure Association (HFA) of the ESC. Eur. J. Heart Fail. 2016, 18, 891-975. [CrossRef]

42. Li, T.; Feng, H.; Xu, Z. A new analytical edge spread function fitting model for modulation transfer function measurement. Chin. Opt. Lett. 2011, 9, 031101. [CrossRef]

43. Rösner, A.; Barbosa, D.; Aarsæther, E.; Kjønås, D.; Schirmer, H.; D’hooge, J. The influence of frame rate on two-dimensional speckle-tracking strain measurements: A study on silico-simulated models and images recorded in patients. Eur. Heart J. Cardiovasc. Imaging 2015, 16, 1137-1147. [CrossRef] [PubMed] 
44. Longère, B.; Chavent, M.H.; Coisne, A.; Gkizas, C.; Pagniez, J.; Simeone, A.; Silvestri, V.; Schmidt, M.; Forman, C.; Montaigne, D.; et al. Single breath-hold compressed sensing real-time cine imaging to assess left ventricular motion in myocardial infraction. Diagn. Interv. Imaging 2020. [CrossRef]

45. Wintersperger, B.J.; Nikolaou, K.; Dietrich, O.; Rieber, J.; Nittka, M.; Reiser, M.F.; Schoenberg, S.O. Single breath-hold real-time cine MR imaging: Improved temporal resolution using generalized autocalibrating partially parallel acquisition (GRAPPA) algorithm. Eur. Radiol. 2003, 13, 1931-1936. [CrossRef] 